\title{
Outcomes of Cystectomy with MAINZ Pouch II and Epispadias Repair in Exstrophy Epispadias Complex in Adults: A Single-centre Experience from Pakistan
}

\author{
Manzoor Hussain, Usman Qamar, Saeed Abidi, Tanzeel Guzdar, Quratul Ain Ghori and Syed Adibul Hasan Rizvi \\ Department of Urology, Sindh Institute of Urology and Transplantation, Karachi, Pakistan
}

\begin{abstract}
Objective: To evaluate surgical outcomes and renal functions after cystectomy + MAINZ Pouch II and epispadias repair as a staged procedure in adult patients with exstrophy epispadias complex (EEC).

Study Design: Descriptive study.

Place and Duration of study: Department of Urology, Sindh Institute of Urology and Transplantation (SIUT), Karachi, from January 2004 to December 2020.

Methodology: A total of 33 patients with EEC were treated. Out of which, 20 underwent cystectomy + MAINZ Pouch II with epispadias repair as a staged procedure. Out of these, 17 had a follow-up period of more than a year and were included in the study. The patients were followed up after 6 weeks of surgery, at 6 months, one year, and at the end of follow-up. The assessed variables included the patients' renal function tests, malignancy potential, morning erections, ejaculations, night emissions, day-and-night-time urinary frequency, and overall happiness of patients at the end of follow-up.

Results: The mean age was $25.1 \pm 7.5$ years and mean follow-up duration was $7.8 \pm 5.2$ years. Postoperatively, there was a rise in blood urea from 27.8 to $35.08 \mathrm{mg} / \mathrm{dl}$ with a concurrent fall in serum bicarbonate from a mean of 23.5 to $20.2 \mathrm{mEq} / \mathrm{dl}$. All patients were continent during the day-time postoperatively; whereas, two patients experienced nocturnal enuresis. All male patients exhibited good erections and ejaculations, but there was persistence of dorsal chordae in $4(23.3 \%)$ patients. All were delighted, happy and pleased with the surgical outcomes and had returned to normal life. No rectal or sigmoid malignancy was observed.
\end{abstract}

Conclusion: In adult EEC patients, cystectomy + MAINZ Pouch II and epispadias repair is safe and effective.

Key Words: Adults, Exstrophy epispadias complex, MAINZ pouch II, Pakistan.

How to cite this article: Hussain M, Qamar U, Abidi S, Guzdar T, Ghori QA, Rizvi SAH. Outcomes of Cystectomy with MAINZ Pouch II and Epispadias Repair in Exstrophy Epispadias Complex in Adults: A Single-centre Experience from Pakistan. J Coll Physicians Surg Pak 2021; 31(10):1191-1195.

\section{INTRODUCTION}

Exstrophy epispadias complex (EEC) is defined as a congenital anomaly, which affects the genitourinary system, gastrointestinal tract, musculoskeletal system, pelvic floor muscles and bony pelvis. The reported incidence ranges between 1 in 10,000 to 1 in 50,000 live births. ${ }^{1}$ In developing countries like Pakistan, there are a few pediatric urology centres, and due to poverty and lack of awareness, many patients do not receive treatment during infancy and present very late in adult life. ${ }^{2}$ Repair of bladder exstrophy begins with closure of bladder and abdominal wall either by the staged repair or by primary repair of exstrophy just afterbirth.

Correspondence to: Dr. Manzoor Hussain, Department of Urology, Sindh Institute of Urology and Transplantation, Karachi, Pakistan

E-mail: hmanzoor2015@gmail.com

Received: May 12, 2021; Revised: September 02, 2021;

Accepted: September 02, 2021

DOI: https://doi.org/10.29271/jcpsp.2021.10.1191
Pelvic osteotomies may be performed at the time of primary closure to deepen their flattened pelvis, close the pubic diastasis and release tension on the abdominal wall. The ideal timing of closure is debatable. Some proponents recommend bladder closure during first 72 hours of life, while others recommend delay in closure of bladder, which can allow surgeons to repair epispadias at the sametime.

There are few reports in the literature on repair in adult patients from developing countries. ${ }^{3}$ Due to all-time incontinence, these patients suffer psychological disturbances, low self-esteem and social isolation and abandonment by the family. Management in adult patients can vary from cystectomy and MAINZ Pouch II and epispadias repair as a single-stage or two-stage repair as done at the study centre. Others have reported cystectomy with cutaneous continent diversion like Indiana pouch; whereas, few surgeons do not do cystectomy and use native bladder i.e., augmentation cystoplasty with bladder neck reconstruction and Mitrofanoff procedure. ${ }^{4}$ The main concern in using rectosigmoid pouch (MAINZ Pouch II) forneobladder formation is deterioration in renal functions and potential for malignancy in the 
future, which can occur with any type of intestinal diversion, or augmentation cystoplasty in $2.5 \%$ cases. ${ }^{5}$ The exact incidence of adult EEC in Pakistan is unknown. There are few case reports in which 12 cases have been reported from Peshawar, ${ }^{6}$ one case report from Karachi, ${ }^{7}$ and another paper reports 10 cases of EEC in pediatric age group from Karachi. ${ }^{8}$ The use of MAINZ Pouch II is very well reported from Germany as a continent diversion after cystectomy in EEC. ${ }^{9}$

With this background, it was decided to look for postoperative renal functions, sexual functions, incontinence, malignancy, surgical complications and health-related well-being in bladder EEC patients, who were treated at this institute by cystectomy and MAINZ Pouch II, followed by epispadias repair as a two-stage procedure. Such cases are rare in adult population, requiring reconstructive urosurgery.

The aim of this study was to evaluate the surgical outcomes and renal functions after cystectomy with MAINZ Pouch II and epispadias repair for EEC in adult patients.

\section{METHODOLOGY}

It was a descriptive study conducted at Department of Urology, Sindh Institute of Urology and Transplantation (SIUT), Karachi, Pakistan. During the years 2004 - 2020, 33 patients of EEC were admitted in the Urology Unit. Patients, who underwent cystectomy and MAINZ Pouch II and epispadias repair as a two-staged procedure, with more than one-year follow-up, were included in this analysis.

Patients who underwent other procedures for EEC, or had less than one year of follow-up were excluded from the study. The criterion for MAINZ Pouch II was competence of anal sphincter with no history of sigmoid colon disease in the past.

Formal approval of this proposal was taken from Institutional Research Advisory Committee and Ethical Committee. Written informed consent was taken from patients who followed up in OPD. Patients were fully counselled regarding benefits and risk of the procedure.

Information regarding age, gender, district of residence, history of renal stone/renal failure was noted on predesigned proforma. Laboratory investigations (serum creatinine, urea, electrolytes) and radiological investigations (ultrasound imaging, $\mathrm{X}$ ray KUB and CT KUB) were done to evaluate for renal size, renal calculi, renal function, surgical and metabolic complications. Post-operative sigmoidoscopy along with rectal biopsy was performed to look for any malignancy at the end of follow-up in December 2020. To assess the sexual function, we asked the patients about morning erections and night emissions, and quality of life was assessed by asking simple questions about overall happiness after operation and follow-up, about continence, and back to work, ability to perform prayers and improvements in self-esteem and confidence.

The surgical technique for cystectomy and MAINZ Pouch II and epispadias repair as a staged procedure was performed as per standard protocol..$^{9,10}$

These patients were kept in the ICU for 24 hours. Oral fluids were started once gut sounds became audible. Total parenteral nutrition was given through subclavian double lumen line for 4 - 5 days. Rectal catheter was removed on the $5^{\text {th }}$ postoperative day and ureteric splints were removed after two weeks, one by one on outpatient basis in the operation theatre. Compressionstockings were used for $4-5$ days. Patient was mobilised after 24 hours and were sent home on the $5^{\text {th }}-6^{\text {th }}$ postoperative day with the instruction to follow up in the outpatient clinic every week for six weeks. Patients were instructed to report in emergency, if there were any complaints of pain and fever.

Data analysis was performed using SPSS version 21. Mean and standard deviation was computed for continuous variables like age, urea, creatinine, sodium, potassium, chloride and bicarbonate; while categorical variables such as gender, ethnicity, previous history of renal stones and renal failure, paralytic ileus, hydronephrosis, uretero-intestinal anastomotic strictures, biopsy findings of malignancy, questions about erectile functions as frequency and percentage. Paired t-test was applied to compare the mean differences or mean change in pre- and postprocedurerenalfunction parameters. $P$-value $<0.05$ was considered as significant.

\section{RESULTS}

Out of 20 patients, only 17 patients, who completed at leastoneyear follow-up, were analysed in this study. However, follow-up ranged from one year to 16 years with mean follow-up of $7.8 \pm$ 5.2 years. There were 16 (94.1\%) males and one (5.9\%) female.

The mean age of patients was $25.1 \pm 6.2$ years with a range from 17 to 36 years. Of all patients, 15 belonged to rural areas and two came from urban areas (Tablel).

Table I: Patient characteristics in 17 patients of exstrophy epispadias complex(EEC).

\begin{tabular}{|l|c|c|}
\hline Patient characteristics & No. & Percentage \\
\hline Male & 16 & $94.1 \%$ \\
\hline Female & 1 & $5.9 \%$ \\
\hline M:F Ratio & $16: 1$ & - \\
\hline Age (range in years) & $17-36$ & - \\
\hline Mean age \pm S.D (years) & $25.18 \pm 6.2$ & - \\
\hline Rural & 15 & $88.2 \%$ \\
\hline Urban & 2 & $11.8 \%$ \\
\hline Marital status: & 1 & $5.9 \%$ \\
Married & 16 & $94.1 \%$ \\
Unmarried & 2 & $11.8 \%$ \\
\hline Associated anomalies: & 1 & $5.9 \%$ \\
Bilateral inguinal hernia & 17 & $100 \%$ \\
Undescended testes & \multicolumn{2}{|c|}{} \\
Pubic symphysis diastasis & \multicolumn{2}{|l|}{ Follow-up period range: 1 - 16 years with mean $7.8 \pm 5.2$ years. } \\
\hline
\end{tabular}

Renal function tests and serum electrolytes were assessed; and ultrasound kidneys was done pre- and postoperatively. Preoperative serum creatinine ranged from 0.85 to 1.4 with mean of $0.89 \pm 0.2$. Preoperative blood urea ranged from $17-41 \mathrm{mg} / \mathrm{dl}$ with a mean value of $27.08 \pm 6.9 \mathrm{mg} / \mathrm{dl}$. 
Table II: Pre- and postoperative renal function test (RFTS) in 17 patients.

\begin{tabular}{|c|c|c|c|c|c|c|}
\hline $\begin{array}{l}\text { Pre- and postoperative renal } \\
\text { function tests (RFTS) }\end{array}$ & $\begin{array}{l}\text { Pre-operative } \\
\text { (RFTS) Range }\end{array}$ & $\begin{array}{c}\text { Pre-operative } \\
\text { (RFTS) } \\
\text { mean } \pm \text { SD }\end{array}$ & $\begin{array}{l}\text { Postoperative } \\
\text { (RFTS) range }\end{array}$ & $\begin{array}{c}\text { Pre-operative } \\
\text { (RFTS) } \\
\text { mean } \pm \text { SD }\end{array}$ & $\begin{array}{l}\text { Pre post mean } \\
\text { difference }\end{array}$ & p-value \\
\hline Blood urea & $17-41$ & $27.08 \pm 6.9$ & $17-54$ & $35.06 \pm 11.1$ & -8.000 & 0.009* \\
\hline Serum creatinine & $0.85-1.4$ & $0.89 \pm 0.2$ & $0.27-1.93$ & $0.88 \pm 0.4$ & 0.01471 & 0.856 \\
\hline Serum sodium & $131-161$ & $140.8 \pm 4.9$ & $130-144$ & $139.24 \pm 4.9$ & -0.0059 & 0.973 \\
\hline Serum potassium & $3.1-6.0$ & $3.9 \pm 0.64$ & $2.9-4.6$ & $3.9 \pm 3.8$ & 1.588 & 0.172 \\
\hline Serum bicarbonate & $17-28$ & $23.24 \pm 3.8$ & $17-21$ & $20.59 \pm 3.5$ & 0.059 & $0.041 *$ \\
\hline Serum chloride & $98-118$ & $106.65 \pm 5.3$ & $98-116$ & $106.59 \pm 4.2$ & 2.647 & 0.965 \\
\hline
\end{tabular}

Table III: Surgical outcomes at last follow up, quality of life, and satisfaction after surgery.

\begin{tabular}{|l|c|c|}
\hline Urinary frequency & Range & Mean \\
\hline Day time & $3-10$ & $5.5 \pm 1.8$ \\
\hline Night time & $1-6$ & $2.7 \pm 1.4$ \\
\hline Surgical outcome at last follow-up & N=17 & Percentage \\
\hline Married & 1 & $(5.9)$ \\
\hline Fertility & 0 & $(0.0)$ \\
\hline Morning erections present & 14 & $(82.4)$ \\
\hline Ejaculation night emission & 13 & $(76.5)$ \\
\hline Incontinence & 0 & $(0.0)$ \\
\hline Nocturnal enuresis & 2 & $(11.8)$ \\
\hline Symptomatic UTI/LUTs & 1 & $(5.9)$ \\
\hline Renal Calculi & 2 & $(11.8)$ \\
\hline Malignancy (rectal/colonic) & 0 & $(0.0)$ \\
\hline Quality of life after surgery & 15 & $(88.2)$ \\
\hline Back to society & 10 & $(58.8)$ \\
\hline Back to work & 15 & $(88.2)$ \\
\hline Ability to perform prayers & 4 & $(23.5)$ \\
\hline Patient satisfaction after surgery & 8 & $(47.1)$ \\
\hline Delighted & 5 & $(29.4)$ \\
\hline Mostly satisfied & 0 & $(0.0)$ \\
\hline Pleased & 0 & $(0.0)$ \\
\hline Unhappy & \multicolumn{2}{|l}{} \\
\hline Mostly dissatisfied & \multicolumn{2}{|l|}{} \\
\hline \multicolumn{2}{|l|}{} \\
\hline \multicolumn{2}{|l|}{} \\
\hline
\end{tabular}

At the end of follow-up, blood urea ranged from $17-54$ $\mathrm{mg} / \mathrm{dl}$ with a mean value of $35.06 \pm 11.1 \mathrm{mg} / \mathrm{dl}$, which despite being significant, was within normal limits. Serum creatinine at last follow-up ranged from $0.27-1.93 \mathrm{mg} / \mathrm{dl}$ with a mean of $0.88 \pm 0.4 \mathrm{mg} / \mathrm{dl}$. There was no difference in pre- and postoperative values of serum creatinine $(p=$ 0.856).

Serum electrolytes pre- and postoperative at the end of follow-up are shown in Table II. However, there has been a significant decrease in serum bicarbonate postoperatively with a mean of $20.59 \pm 3.5 \mathrm{mEq} / \mathrm{dl}$ from $23.5 \pm 3.2 \mathrm{mEq} / \mathrm{dl}$ preoperatively. This value was also within limits and no patient was symptomatic (Table II).

Native bladder biopsies after cystectomy were available in all 17 patients, which showed cystitis cystica glandularis in $15(88.2 \%)$ specimens and squamous metaplasia in 2 (11.8\%) specimens. In the background of above changes, only one patient (5.9\%) had high grade sarcomatoid carcinoma of native exstrophy bladder.

Analysis of postoperative surgical complications exhibited the occurrence of paralytic ileus in one $(5.8 \%)$, wound dehiscence in three $(17.6 \%)$, incisional hernia in two $(11.7 \%)$, and persistence of mild dorsal chordae in four (23.5\%) patients. No postoperative mortality was seen in this series. Ultrasound findings in postoperative period included renal calculi in two (11.8\%) and unilateral small kidney due to pyelonephritis in three $(17.6 \%)$ cases; none had hydronephrosis. None of the patients in this series required either balloon dilatation or ureteric reimplantation or any redo surgery. All 17 patients were followed in OPD for urinary continence following cystectomy and MAINZ Pouch II. All were found continent during the day and night time except for two patients, who complained of nocturnal enuresis. Mean voiding frequency during the daytime was $5.5 \pm 1.8$ times and $2.7 \pm 1.4$ during the night time. Only one patient complained of frequent diarrhoea after four years of surgery (Table III).

On analysis of surgical outcomes, the authors found that only one patient had gotten married after surgery with positive history of erections and ejaculations. The rest of the male patients, who were not married, also gave positive history regarding morning erections and ejaculations. After successful repair, patients showed great satisfaction on the quality of life about body image. About 10 (58.8\%) patients were back to work and 15 (88.2\%) back to society and were attending social gatherings with friends. All patients were delighted, happy or pleased after surgery.

Malignant potential after MAINZ Pouch II was assessed at the end of follow-up by colonoscopy and biopsy from suspicious areas. Biopsy results were available in 17 patients, which showed no malignancy. On rectal biopsy, only mild non-specific colitis was seen in $15 / 17$ patients; whereas, the remaining two rectal biopsies were normal.

\section{DISCUSSION}

In the course of the last 16 years (2004 - 2020), 33 patients with EEC were admitted for management at this institute. Out of 33, twenty underwent cystectomy + MAINZ Pouch II + epispadias repair as a staged procedure. Here, the authors analyse 
and discuss only 17 patients who had completed at least one year of follow-up.

There are only three paediatric urology units in Pakistan for more than 207 million population; and only one centre, where antenatal diagnosis of EEC with other congenital urological anomalies is being diagnosed. ${ }^{2}$ Due to lack of facility, poverty and illiteracy, the authors come across this condition in adults at the rate of an average two cases per year. This point is also proven from the data on patient characteristics in this series where $15(88.2 \%)$ of the patients were found to be poor and illiterate and belonged to rural areas.

The literature on this topic from Pakistan is rare except for few case reports. ${ }^{7}$ The reason cystectomy + MAINZ Pouch II surgery was selected for this series is that literature reviews show a high potential of malignancy changes (around 700 times more than normal population) in patients with EEC, when it is exposed to air and left untreated. ${ }^{11,12}$ This point is also proven in this data of 17 patients, who were admitted and managed with various forms of operation. Only one $(5.9 \%)$ of native bladder biopsies showed malignancy reported as sarcomatoid carcinoma. Other native bladder biopsy reports showed cystitis cystica glandularis and squamous metaplasia or a combination of the two as the commonest histopathological findings, which are pre-malignant conditions. The potential for malignancy in native bladder in EEC patients support the point of removing these bladders in adults. ${ }^{13}$

In this series, the main concern of the patients was incontinence of urine that affected their quality of life very badly. Their families abandoned many patients due to smell of urine. Such patients could not do any of their daily activities properly. They could not go to work or places of worship and hence, could not perform prayers. The present data showed that most of the patients were illiterate because they could not attend school; and became jobless as they grew up were not able to get a job due to incontinence of urine. Most of the patients came from rural areas of the country where availability of catheters is a problem, so clean intermittent self catheterisation (CISC) is not costeffective and bothersome for some patients. Therefore, cystectomy and MAINZ Pouch II relieved them of their incapability to perform daily activities. As there was no need for CISC, no need of wearing any appliance for collection of urine and no stone formation in rectosigmoid pouch. This operation became the first choice of patients in contrast to other procedures, including cystectomy and ileal conduit and preservation of the bladder with augmentation cystoplasty, bladder neck resection and Mitrofanoff procedure. However, Kiran et al. reported primary closure and continent cutaneous diversion after cystectomy or ileal conduit as an operation in patients coming from remote areas and poor backgrounds. $^{14}$

In adult patients, the important outcome parameters in management are incontinence, nocturnal enuresis, renal function preservation, sexual and fertility function, satisfaction and overall social well being. The rehabilitation back to society after operation is another parameter worth mentioning in this group of patients. Cosmesis, correction of dorsal chordae, erections and ejaculation are other outcome parameters in successful epispadias repairs, which were considered in these patients. In the long-term follow-up, metabolic abnormalities (hyperchloremic acidosis), deterioration of renal functions and malignancies occurring at the junction of uretero-intestinal anastomosis is an another yet primary concern. In the paper, the authors have focused on these aspects.

On comparing pre- and postoperative renal function tests such as serum creatinine, blood urea and serum electrolytes remained same as before surgery, except for low serum bicarbonate and high blood urea at the end of follow-up. These metabolic findings were significant; but were still within normal limits, which were treated by giving sodium bicarbonate tablets and high citrus fruits and increasing fluid intake. Gerharz et al., however, reported the occurrence of hyperchloraemic acidosis in two of 34 cases, but both of them were mild and treated medically. ${ }^{15}$

All 17 patients were found to be continent during the entire day with only two patients complaining of nocturnal enuresis occasionally. This complaint was managed by advising these two patients to reduce the amount of fluid intake including water, milk or tea before going to bed and to keep a buzzer alarm that will help them to wake up at least once or twice during the night for voiding in order to prevent nocturnal enuresis. These practices not only helped to improve nocturnal enuresis but also improved their quality of life. The excellent day and night-time continence rate in this series is in agreement with the findings of Delia et al. from Germany. ${ }^{9}$

The main goal of this study was to satisfy the patients; and to have achieved this, is a major goal for the team. This surgery helped the patients return to society and they can live their lives without any fear of abandonment and stigma. All of them returned to work and places of worship - this means all patients were rehabilitated to useful life and became useful members of the society. There was no complaint of pouch stone formation, mucus secretion or need for intermittent catheterisation; thus, making this operation a preferable option for treating EEC in a resource-limited country like Pakistan.

The technique of detubularisation of rectosigmoid segment and antireflexing ureteric implantation by using submucosal tunnel and putting ureteric stents (10 FR long feeding tubes) for two weeks, to make the spherical reservoir with no complications of ureteric obstruction in this series and maintenance of renal functions is also a good achievement in this series. Sexual and fertility functions in only one female patient could not be discussed in this paper due to small number of patients (only one of all cases), who remained unmarried. Mathhew et al. addressed the issue of urogynecology and obstetrics in series of 83 women who were treated in infancy and childhood and became adults. He reported their term pregnancies in 8 female patients and described occurrence of uterine prolapse in this series. ${ }^{16}$ 
The epispadias repair by modified Cantwell-Ranseley technique was done after six months of MAINZ Pouch II. All patients were happy with cosmesis, erections and ejaculations, except for those who had persistence of dorsal chordae and required re-do operation. To-date, the authors have not yet seen any malignancies in MAINZ Pouch II in this series, but the risk of colonic carcinoma has been estimated to be about $5 \%$ after ureterosigmoidostomy. ${ }^{17}$ Hence, screening of all patients with urinary diversion is mandatory after five years of surgery, followed by the same every year. These patients need life-long follow-up for deterioration of renal functions and malignancy of rectal pouch.

\section{CONCLUSION}

This study concludes that cystectomy + MAINZ Pouch II and staged epispadias repair provide good results, very high patient satisfaction, and rehabilitation back to society. The renal functions remained stable and no case of recto-sigmoid malignancy was reported. Cosmetic results after genital reconstruction and epispadias repair were satisfactory.

\section{ETHICAL APPROVAL:}

This research was approved by the Ethical Review Committee (ERC) of SIUT, Karachi, Pakistan.

\section{PATIENTS' CONSENT:}

Written informed consents were obtained from all patients before surgery regarding the procedure and publication of the results of this work.

\section{CONFLICT OF INTEREST:}

The authors declared no conflict of interest.

\section{AUTHORS' CONTRIBUTION:}

MH, QAG: Conception, data collection, data analysis, results, primary drafting and final approval.

UQ: Data collection, results, discussion and literature review.

SA, TG: Results, discussion and literature review.

SAHR: Final approval.

\section{REFERENCES}

1. Feldkamp ML, Botto LD, Amar E, Bakker MK, Bermejo-Sánchez E, Bianca S, et al. Cloacal exstrophy: An epidemiologic study from the international clearinghouse for birth defects surveillance and research. Am J Med Genet C Semin Med Genet 2011; 157C(4):333-43. doi: 10.1002/ajmg.c. 30317.

2. Sultan S. Evolution of paediatric urology at sindh institute of urology and transplantation. Front Pediatr 2014; 8:2:88. doi: 10.3389/fped.2014.00088.

3. Mansour AM, Sarhan OM, Helmy TE, Awad B, Dawaba MS, Ghali AM. Management of bladder exstrophy epispadias complex in adults: + abdominal closure possible without osteotomy? World J Urol 2010; 28(2):199-204. doi: 10. 1007/s00345-009-0436-7.

4. Jana A, Maiti K, Mondal TK, Majhi TK. Management of exstrophy epispadias complex in adolescents and adults. Afr J Urol 2017; 23:100-4.

5. Lakshman T, Patel J, Shenot P, Isenberg G. Signet ring cell cancer of colon 48 years after ureterosigmoidostomy, treated with low anterior resection and continent urinary diversion. ACS Case Rev Surg 2017; 1:1-4.

6. Khan M, Muhammad S, Shah FO, Hadi A, Kahim M. An experience with continent urinary diversion. J Surg Pakistan 2016; 21:67-70.

7. Irfan O, Lodiwala ZF, Zaidi Z. Long-term follow-up of Exstrophy Epispadias Complex from a lower-middle income country: A Case report and review of literature. Cureus 2020; 12(4):e7723. doi: 10.7759/cureus. 7723.

8. Sultan S, Hussain I, Umer SA, Saulat S, Naqvi SA, Rizvi SAH. Clean Intermittent catheterisation in children through a continent catheterisable channel: A developing country experience. J Urol 2008; 180(4 Suppl): 1852-5. doi: 10.1016/j.juro.2008.03.118.

9. D'elia G, Pahernik S, Fisch M, Hohenfellner R, Thuroff JW. Mainz Pouch II Technique: 10 Years' Experience. BrJ Urol Int 200; 93(7):1037-42. doi: 10.1111/j.1464-410X.2003. 04777.x.

10. Baird AD, Gearhart JP, Mathews RI. Applications of the modified Cantwell-Ransley epispadias repair in the exstrophy-epispadias complex. J Pediatr Urol 2005; 1(5):331-6. doi: 10.1016/j.jpurol.2005.02.003.

11. Xiong W, Peng R, Zhu L, Zhong Z. Bladder exstrophy epispadias complex with adenocarcinoma in an adult patient: $A$ case report. Exp Ther Med 2015; 10(6):2194-6. doi: 10. 3892/etm.2015.2793.

12. Smeulders $N$, Woodhouse $C R$. Neoplasia in adult exstrophy patients. Br J Urol Int 2001; 87(7):623-8. doi: 10.1046/j. 1464-410x.2001.02136.x.

13. Shoukry Al, Shoukry I. Management of bladder exstrophy in adulthood: Report of 5 cases. J Pediatr Urol 2013; 9(5): 575-8. doi: 10.1016/j.jpurol.2012.06.004.

14. Kiran PS, Panaiyadiyan S, Singh P, Nayak B, Nayyar R, Seth A. Management of untreated classical bladder exstrophy in adults: A single institutional experience. Urology 2020; 146:293-8. doi: 10.1016/j.urology.2020.09.009.

15. Gerharz EW, Köhl UN, Weingärtner K, Kleinhans BJ, Melekos $\mathrm{MD}$, Riedmiller $\mathrm{H}$. Experience with the Mainz modification of ureterosigmoidostomy. Br J Surg 1998; 85(11):1512-6. doi: 10.1046/j.1365-2168.1998.00904.X.

16. Matthews RI, Bau M, Gearhart JP. Urogynecological and obstetric issues in women with the exstrophy epispadias complex. Br J Urol Int 2003; 91(9):845-9. doi: 10.1046/j. 1464-410x.2003.04244.x.

17. Ragu R, Meurette G, Kim M, Le Normand L, Lehur PA. Carcinoma arising in enteric diversion or rectal neobladder for bladder exstrophy. Tech Coloproctol 2016; 20(11):745-752. doi: 10.1007/s10151-016-1519-2. 